\title{
Highly Sensitive Pesticide Detection using Electrochemically Pre- pared Silver-Gum Arabic Nanocluster-based SERS Substrates
}

\author{
Yuqing Yang, Alan O’Riordan, Pierre Lovera \\ Nanotechnology Group, Tyndall National Institute - University College Cork, Dyke Parade, Cork, Ireland \\ KEYWORDS Surface Enhanced Raman Spectroscopy, Ag-Gum Arabic SERS substrate, electrochemical deposition, 2,4-di- \\ chlorophenoxyacetic acid (2,4-D) pesticide detection.
}

\begin{abstract}
Surface Enhanced Raman is a promising analytical method for harmful pesticides detection. Herein, we demonstrate a rapid method to directly monitor 2,4-D pesticide in water without the need of sample pre-treatment by using Silver-Gum Arabic (Ag-GA) electrochemically co-deposited SERS substrates. The facile electrochemical synthesis of the large-area (40 $\mu \mathrm{m} \times 20 \mu \mathrm{m})$ SERS substrates needs only 10 seconds, with $2 \mu \mathrm{L} \mathrm{AgNO}_{3}$ and Gum Arabic mixed solution. Different morphologies including AgGA spherical nanoparticles and nano dendrites were synthesized by tuning the deposition parameters. This study therefore offers a novel way to fabricate SERS substrates for rapid determination of 2,4-D pesticides down to $1 \mathrm{pM}$ in deionized water and $0.15 \mathrm{nM}$ in commercial bottled and also river water samples.
\end{abstract}

With the world population predicted to reach 9.8 billion by 2050 , it is necessary to increase food production accordingly. ${ }^{1}$ Unfortunately, crops are under a number of threats from insects, diseases or competing weeds. Thus, pesticides are widely used to protect crops and increase yield at the same time. Amongst these, 2,4-dichlorophenoxyacetic acid (2,4-D, $\left.\mathrm{C}_{8} \mathrm{H}_{6} \mathrm{Cl}_{2} \mathrm{O}_{3}\right)$ is a phenoxy herbicide that is extensively applied in agriculture to control weeds in corn and grain field. ${ }^{2}$ Unfortunately, there is growing evidence that 2,4-D is harmful both to human health and the environment. ${ }^{3-4}$ To prevent or minimize negative impacts from the use of pesticides, the European Union has regulated their use and has provided strict guidelines for their application. For example, a maximum residue limits (MRL) of 0.1 ppb (parts per billion) for individual pesticide has been established. ${ }^{5}$ However, despite these precautions, pesticides can still leach from the ground into water and run off in nearby water bodies. $^{6}$ 2,4-D in particular has good solubility in water and traces above the MRL have been found in river and drinking water. ${ }^{7}$ To this end, it is necessary to monitor $2,4-\mathrm{D}$ pesticides and possibly prevent pollution events. The most common method currently used to detect 2,4-D in real environment is by applying chromatography to purify the small molecule, followed by mass spectrometry (HLC-MS) to qualitatively identify the herbicides. ${ }^{8}$ Unfortunately, this method is time-consuming and requires high-end equipment operated by skilled personnel in dedicated laboratories. To tackle this challenge, numerous of methods have been investigated in recent years for simpler 2,4-D detection, including platforms based on electrochemistry,${ }^{9}$ fluorescence, ${ }^{10}$ chemiluminescence, ${ }^{11}$ etc. Amongst them, optical detection based on surface enhanced Raman spectroscopy (SERS) has shown tremendous potential. ${ }^{8,12}$

Surface Enhanced Raman Spectroscopy is mostly based on the enhanced Raman signal by metallic nano structures that can generate strong electromagnetic field with the excitation at certain laser wavelength. ${ }^{13}$ SERS is an ultra-sensitive technology that is capable of providing a spectral fingerprint of the analytes under investigation. The magnitude of the SERS enhancement depends on the morphology, the size of the metallic nano particles, the gap size between the metallic nano particles, the measurement environment, as well as the wavelength of the laser excitation source. ${ }^{14-17}$ Because of the simplicity of operation and sensitivity in detection, SERS has been used for trace detection of 2,4-D pesticides ${ }^{18}$ including: SERS substrates based on hollow Au@Ag nano-snowflake particles functionalized with 4MBA/2,4-D antigen, ${ }^{12}$ citrate functionalized AgNPs on cellulose paper, ${ }^{19}$ silver NPs with molecular imprint polymers (MIP) ${ }^{20}$ highly roughened surface flower-like Ag NPs. ${ }^{21}$ Particularly, SERS substrates based on well-ordered AuNPs inside mesostructured silica channels ${ }^{22}$ have been used to detect 2,4$\mathrm{D}$, with the limit of detection (LOD) down to $0.79 \mathrm{ppt}$ (parts per trillion) achieved. ${ }^{22}$ These methods require either (i) an incubation time of more than $1 \mathrm{~h}$, (ii) a pre-treatment step by mixing the solution with ethanol, or (iii) waiting time to allow 2,4-D solution to dry on the SERS substrate. To rapid determine pesticides such as 2,4-D directly in water samples without any pretreatment remains a key challenge.

SERS substrates are commonly fabricated through chemical synthesis, ${ }^{23-24}$ electron beam (E-beam) lithography, ${ }^{25}$ or templating method. ${ }^{26-28}$ These methods are either time consuming or involve complex steps. High throughput, low cost and reproducible fabrication of SERS substrates is still elusive.

Herein, we report on the rapid and reproducible fabrication of Silver-Gum Arabic (Ag-GA) SERS substrates by electrochemical deposition. The fabrication method produces large area SERS substrate $(40 \mu \mathrm{m} \times 20 \mu \mathrm{m})$ and requires less than 10 
s, with $2 \mu \mathrm{L}$ of a non-toxic solvent. This is a time-saving, environment friendly, high throughput and scalable method in comparison to the other ways of fabricating the SERS substrate. The fabricated samples are used for herbicide 2,4-D detection directly in aqueous solutions, with a detection limit down to $1 \mathrm{pM}$ reported without the need for sample preparation.

Gum Arabic was selected as it is a branched hetero-polysaccharide containing protein and carbohydrate groups. ${ }^{29-30}$ It has been used to act as a biopolymer for removal of pesticides in polluted water bodies. ${ }^{31-32}$ Gum Arabic has been used for green chemical synthesis of silver nano particles. ${ }^{33-35}$ During this process, Gum Arabic acts as a reducing agent to reduce silver ions, then Gum Arabic forms a complex with Ag atoms, subsequently forming Ag-GA NPs. ${ }^{35}$ In this work, an electrochemical deposition method was used to fabricate Ag-GA SERS substrates. The size and morphology of the silver nanoparticles was tailored by controlling electrochemical deposition parameters such as deposition time, deposition voltage, and the precursor concentration. During the electrochemical deposition process, silver ions are electrochemically reduced, then combine with Gum Arabic monomers to form a Ag-GA nanoclusters complex, which deposit on the chip surface in the vicinity of the electrodes. The electrochemical approach greatly accelerated $\mathrm{Ag}^{+} \rightarrow \mathrm{Ag}$ process compared to the natural reducing process by the Gum Arabic alone. ${ }^{34}$ In addition, tailoring deposition parameters, enables not only the size and morphology of the AgGA nanoclusters, but also the regular distributed gap pattern in between the nanoclusters, to be engineered. 


\section{EXPERIMENTAL SECTION}

\section{Materials}

$\mathrm{AgNO}_{3}$ was purchased from Sigma-Aldrich ( Ireland ). Gum Arabic was purchased from both Sigma Aldrich and from Amazon.co.uk. The commercial mineral water was purchased from local shopping market. The real river sample was collected from the local river Lee, Cork, Ireland. Samples were prepared using ultra-pure Mili-Q water $\left(18.2 \mathrm{M} \Omega \cdot \mathrm{cm}^{-1}\right.$, Mili-Q). Electrochemical depositions were undertaken with an Ivium pocketSTAT handheld potentiostat operating under computer control.

\section{Microelectrode Fabrication on $\mathrm{Si} / \mathrm{SiO}_{2}$ chip}

SERS sensors were fabricated on four-inch silicon wafer substrates with a $300 \mathrm{~nm}$ layer of thermally grown silicon dioxide as previously described. ${ }^{36}$ Briefly, the working electrodes were patterned using photolithography and physical vapor deposition (PVD) (50 nm of Au, with $10 \mathrm{~nm}$ of Ti adhesion layer) followed by a lift-off step. A second optical lithographic and metal deposition process ( $\mathrm{Au} 100 \mathrm{~nm} / \mathrm{Ti} 10 \mathrm{~nm}$ ) was undertaken to define micro-SD pin-out, interconnection tracks, as well as the on-chip counter electrode $(500 \mu \mathrm{m}$ wide $\times 6 \mathrm{~mm}$ long). A third lithographic step was employed to define the Pt (Pt $100 \mathrm{~nm} / \mathrm{Ti} 10$ $\mathrm{nm})$ on-chip reference electrode $(500 \mu \mathrm{m}$ wide $\times 6 \mathrm{~mm}$ long$)$. Finally, $500 \mathrm{~nm}$ of Plasma-enhanced chemical vapor deposition (PECVD) $\mathrm{Si}_{3} \mathrm{~N}_{4}$ was blanket deposited on the whole wafer, the openings were over the working/counter/reference electrodes and the electrical contacts were defined by lithography and dry etching process. See Figure S1 in SI.

\section{Synthesis of Gum Arabic Coated Ag (Ag-GA) nanoclus- ters}

The SERS substrates were prepared using different concentrations of Gum Arabic and $5 \mathrm{mM} \mathrm{AgNO}_{3}$ solutions. Gum Arabic solutions were mixed by hand with a glass rod at ambient temperature. For the electrochemical deposition, the two solutions were mixed with 1:1 ratio and a small aliquot $(2 \mu \mathrm{L})$ was dropped on a chip surface to cover the working electrode, the on chip pseudo reference and counter electrodes. $\mathrm{AgNO}_{3}$ and Gum Arabic solutions were mixed just before the SERS substrate fabrication, as given enough time, $\mathrm{AgNO}_{3}$ and Gum Arabic would chemically react to form Ag nanoparticles. Electrochemical deposition was undertaken with an Ivium pocketSTAT handheld potentiostat using the parameters presented in Table 1 (below). Ag-GA substrates were then rinsed in DI water and dried under a nitrogen flow for several seconds.

\section{Microscopic and SEM characterization}

Microscopic images of the fabricated SERS substrates were acquired with Renishaw Raman system with a 50 x (NA 0.75) objective. SEM characterization was undertaken with a FEI Quanta 650, the accelerate voltage used was $10 \mathrm{KV}$, the spot size was $2.5 \mathrm{~mm}$.

\section{Raman measurements}

Raman spectra were acquired with a Renishaw InVia microscope equipped with a $514 \mathrm{~nm} \mathrm{Ag}$ ion laser. For the measurements, SERS substrates were placed in a dedicated holder, shown in SI Figure S2 (C-D). A $10 \mu \mathrm{L}$ aliquot of 2,4-D solution was dropped over Ag-GA SERS substrate and covered with a cover glass slide. All measurements were acquired using a 20x lens (NA 0.4). All the spectra presented herein were averaged from ten measurements obtained at different random points on the substrate except for the reproducibility study where 25 measurements were employed. All spectra were obtained using a laser power of $0.07 \mathrm{~mW}$ and an integration time of $10 \mathrm{~s}$.

\section{Data Analysis}

All spectra were corrected using the baseline subtraction algorithm, ${ }^{37}$ the false colour map was performed by Matlab. The baseline subtraction process was shown in Figure S3 in SI. The linear fitting was done by Origin ${ }^{\mathrm{TM}}$ (OriginPro 2016).

\section{RESULTS AND DISCUSSION \\ Ag-GA SERS substrate Fabrication}

A microband sensor is presented in Figure 1. The sensor chips were originally developed for electrochemical based sensing applications. ${ }^{36,38}$ The detailed chip circuit design is presented in SI Figure S2 (E), the chips had a micro-SD pin-out for easy electrical plug-and-play connectivity to the potentiostat (see SI Figure S2 (A-B)). Each chip had six working electrodes that may be individually addressed. In the present work, the electrodes employed were single Au micro bands ( $1 \mu \mathrm{m}$ wide, 45 $\mu \mathrm{m}$ long, $50 \mathrm{~nm}$ height). These were selected as the high current densities, observed at ultra microelectrodes, enabled the formation of nanoclusters emanating away from the band. A small aliquot - for example, only $2 \mu \mathrm{L}$ - of the mixed $\mathrm{GA} / \mathrm{AgNO}_{3}$ solution was required for one SERS substrate fabrication on the chip. Considering this electrodeposition process took only 10 seconds, the present approach was rapid, low cost, scalable and environmentally friendly. Figure 1 (B) shows a SEM image of a SERS structures electrodeposited on a micro-band electrode. The nanoclusters were strongly attached to the chip surface, were stable and allowed hundreds of Raman measurements to be undertake without any degradation of the substrate being observed. 

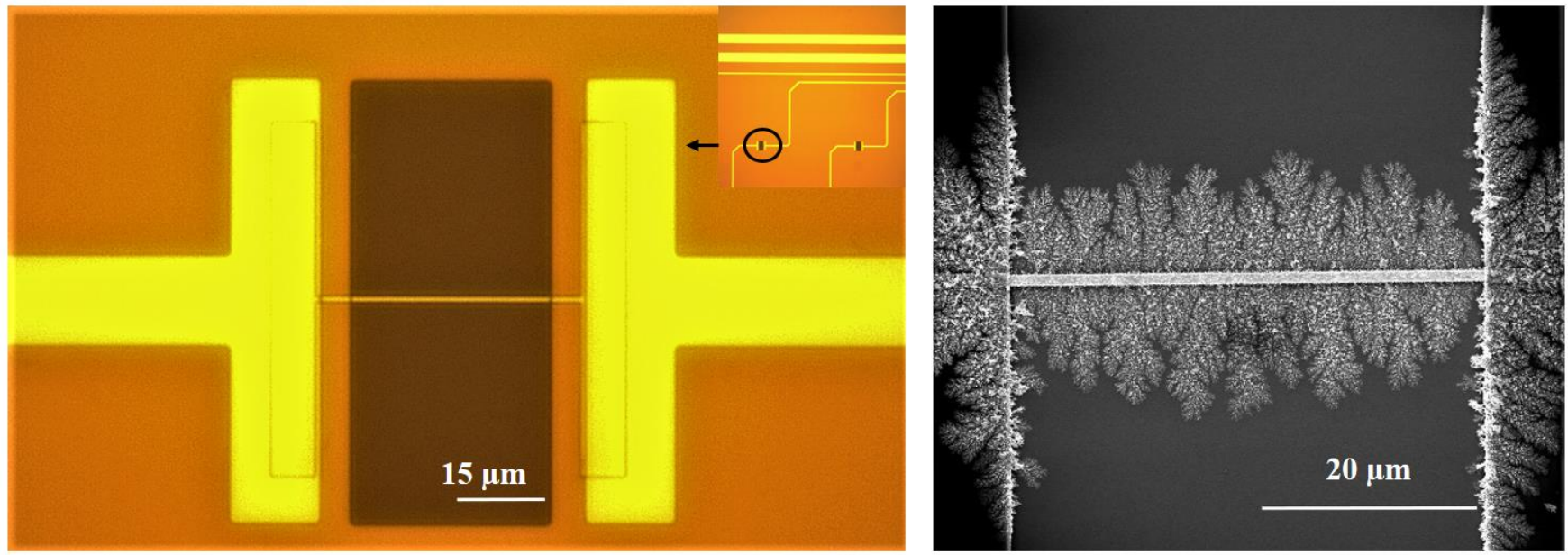

Figure 1 (A) Microscope image (25 x): micro-band working electrode on the chip (20 x under microscope); Insert: three electrode system for electrochemical deposition (2.5 x under microscope). (B) SEM image for a SERS substrate.

\section{Electrodeposition process and sample characterization}

Figure 2 (A - D) show SEM images of four Ag-GA nanostructures prepared using different experimental parameters as described in Table 1. The relationship between the deposition current (nA) and time (s) is shown in SI Figure S4 (A). The deposition current shows a linear relationship with deposition time, and the maximum current for the four SERS substrates were $70 \mathrm{nA}, 60 \mathrm{nA}, 90 \mathrm{nA}, 120 \mathrm{nA}$, respectively. The false colour map in Figure 2 (A - D) show different SERS substrate structures. False colour maps clearly indicated the nanocluster space distribution across the surfaces, as well as the 3-D nature of the deposited nanocluster structures. The colour bar indicates the distance of the nanocluster from $\mathrm{SiO}_{2} / \mathrm{Si}$ substrate, the deep blue colour (colour bar value at $0 \mathrm{~nm}$ ) is $\mathrm{SiO}_{2} / \mathrm{Si}$ reference surface, the nanoclusters that present light green colour are around $50 \mathrm{~nm}$ in height, which is around the same height as the working electrode. The higher the number in the colour bar, the further the nanoclusters are away from $\mathrm{SiO}_{2} / \mathrm{Si}$ substrate. The nanoclusters that showed a yellow colour are $\sim 100$ $\mathrm{nm}$ away from $\mathrm{SiO}_{2} / \mathrm{Si}$ surface) while the nanoclusters that showed a red colour were more than $100 \mathrm{~nm}$ away from the $\mathrm{SiO}_{2} / \mathrm{Si}$ reference surface. Original SEM images are presented in SI Figures S5 (A-D), optical images are also available (see Figure S4 (C)). Figures $2(\mathrm{E}-\mathrm{H})$ show high-resolution SEM images of nanoclusters for the four structures. Ag-GA nano dendrite structures formed in figure 2 (E-G) labelled structure $S_{1}$, $\mathrm{S}_{2}, \mathrm{~S}_{3}$, while round-shape Ag-GA nano particles growth was observed in Figure $2(\mathrm{H})$, labelled structure $\mathrm{S}_{4}$. The size of the AgGA nano particles in structure $S_{4}$ were estimated to be in the range of 50 - $70 \mathrm{~nm}$, see SI Figure S6. For structures $S_{1}$ and $S_{3}$, Ag-GA nano dendrites growth on the surface of $\mathrm{SiO}_{2}$ was observed to be orthogonal to the chips surface. See also SEM image tilted at $35^{\circ}$ in SI Figure $\mathrm{S} 7$ that showed the isolated growth of the nanoclusters on $\mathrm{SiO}_{2}$. By comparison, for structure $\mathrm{S}_{2}$, the Ag-GA nano dendrites growth along the Au electrode and stacked together orthogonal to the chip surface.
Nanoclusters and nanogap formation may be explained by the interplay between mass transport (diffusion and electron migration) of Ag ions and passivation by Gum Arabic. After mixing with Gum Arabic, the resistance of the mixed solution is higher than $\mathrm{AgNO}_{3}$ alone. This was inferred by the recordings of the electrical current when using solutions with and without Gum Arabic, see SI Figure S4 (A) and Figure S8 (A). Using the same deposition parameter as $\mathrm{S}_{4}(-0.2 \mathrm{~V}, 2 \mathrm{~s} ;-0.8 \mathrm{~V}, 8 \mathrm{~s})$, the maximum deposition current was about $2 \mu \mathrm{A}$ without Gum Arabic, while it was around $120 \mathrm{nA}$ after mixing $\mathrm{AgNO}_{3}$ with Gum Arabic. The reduction of $\mathrm{Ag}$ ions to $\mathrm{Ag}$ atoms reaction happened on the substrate emanating away from the electrodes due to the strong electric field. The Ag-GA complex formed immediately and stacked on the $\mathrm{SiO}_{2} / \mathrm{Si}$ substrate. The formation of Ag-GA nanoclusters further affected the electric field distribution and helped the formation of nanogaps in between the nanoclusters. ${ }^{39}$ As different concentrations of Gum Arabic solutions had different resistances, it was observed that the resistance increased as the concentration of Gum Arabic increased within certain Gum Arabic concentrations. ${ }^{40}$ According to Ohm's law, $\mathrm{J}=\sigma \mathrm{E}$, where $\mathrm{J}$ is the current density, $\sigma$ is the electrical conductivity, $\mathrm{E}$ is electric field, different concentrations of Gum Arabic solutions resulted in different electrical conductivity for the mixed silver precursor solutions, which further resulted in different current densities in the electrochemical system. Thus, there were different Ag-GA nanostructures formed with different Gum Arabic concentrations when the other deposition parameters were maintained (see structure 1, 3, 4 and SI Figure S9 (AE).). The applied voltage also affected the growth pattern and growth direction of the Ag-GA nano particles (see structure 1, 2 and SI Figure S9 (M-O). The detailed discussion is presented in SI. In conclusion, by adjusting the concentration of $\mathrm{AgNO}_{3}$ and Gum Arabic in the solution as well as the electrochemical deposition parameters such as applied voltage and deposition time, it is possible to engineer the size and morphology of the Ag-GA nano structures and the nano gap patterns in between 
the nano clusters. The high density of nanostructures and nano gaps made these substrates good candidates for SERS applications.

Table 1 Electrochemical deposition parameters for the 4 structures

\begin{tabular}{|l|c|l|l|c|}
\hline SERS substrate & {$\left[\mathrm{AgNO}_{3}\right]$} & [Gum Arabic] & Applied potential and deposition time & $\begin{array}{c}\text { Maximum current } \\
(\mathrm{nA})\end{array}$ \\
\hline $\mathrm{S}_{1}$ & $5 \mathrm{mM}$ & $2 \mathrm{~g} / \mathrm{L}$ & $\begin{array}{l}\text { Step 1: }-0.2 \mathrm{~V}, 2 \mathrm{~s} ; \\
\text { Step 2: }-0.8 \mathrm{~V}, 8 \mathrm{~s} .\end{array}$ & 70 \\
\hline $\mathrm{S}_{2}$ & $5 \mathrm{mM}$ & $2 \mathrm{~g} / \mathrm{L}$ & $\begin{array}{l}\text { Step 1: }-0.2 \mathrm{~V}, 2 \mathrm{~s} ; \\
\text { Step 2: }-0.5 \mathrm{~V}, 8 \mathrm{~s} ; \\
\text { Step 3: }-0.6 \mathrm{~V}, 2 \mathrm{~s} .\end{array}$ & 60 \\
& & & $\begin{array}{l}\text { Step 1: }-0.2 \mathrm{~V}, 2 \mathrm{~s} ; \\
\text { Step 2: }-0.8 \mathrm{~V}, 8 \mathrm{~s} .\end{array}$ & 90 \\
\hline $\mathrm{S}_{3}$ & $5 \mathrm{mM}$ & $1 \mathrm{~g} / \mathrm{L}$ & $\begin{array}{l}\text { Step 1: }-0.2 \mathrm{~V}, 2 \mathrm{~s} ; \\
\text { Step 2: }-0.8 \mathrm{~V}, 8 \mathrm{~s} .\end{array}$ & 120 \\
\hline $\mathrm{S}_{4}$ & $5 \mathrm{mM}$ & $0.5 \mathrm{~g} / \mathrm{L}$ & & \\
\hline
\end{tabular}
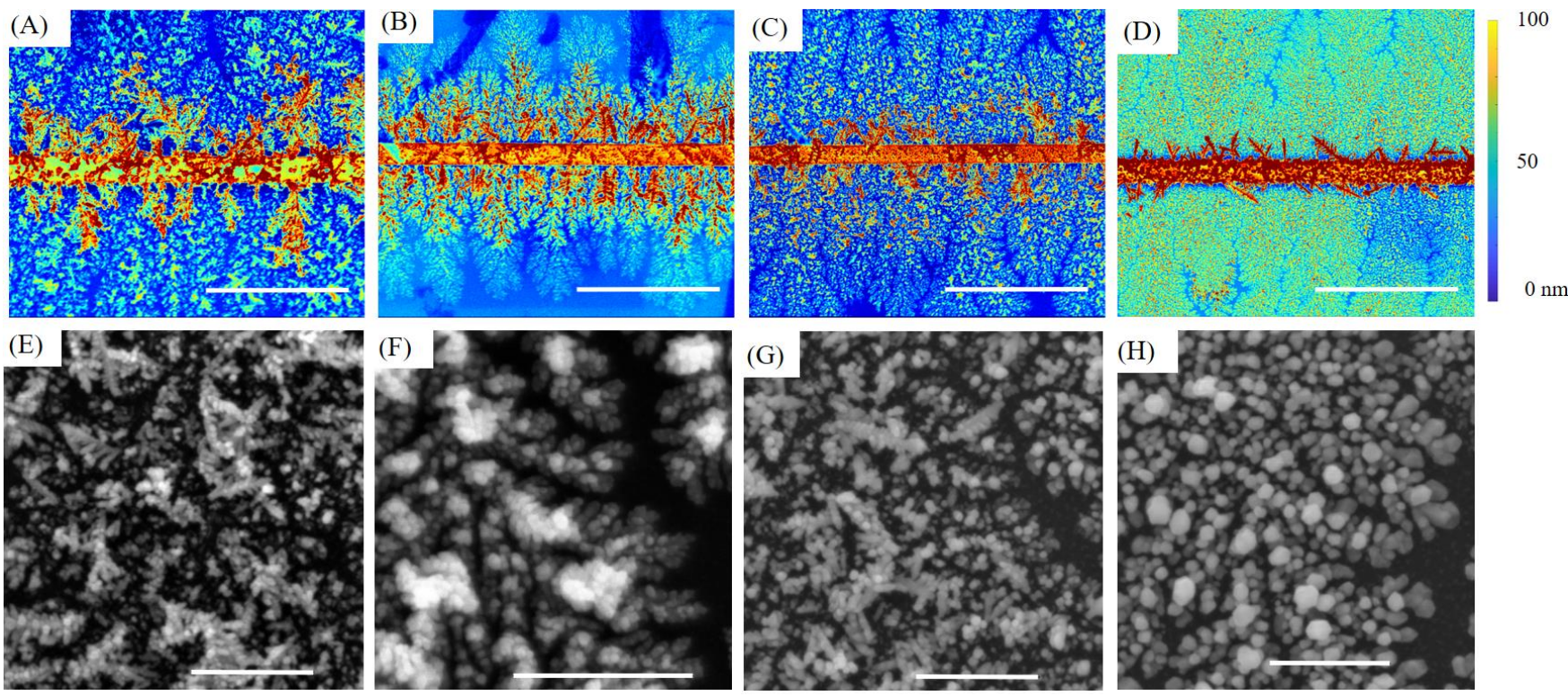

Figure 2 (A - D) False colour map from SEM image for structure one to structure four by Matlab, scale bar $5 \mu \mathrm{m}$; the color bar at $0 \mathrm{~nm}$ is the $\mathrm{SiO}_{2} / \mathrm{Si}$ reference surface. $(\mathrm{E}-\mathrm{H}) \mathrm{SEM}$ image of nano structure for structure one to structure four. Scale bars; $500 \mathrm{~nm}$.

\section{SERS detection of 2,4-D pesticide} Initial Raman detection

Gum Arabic has previously been used to interact with pesticides $^{31}$ and is known to attract an analyte in close proximity to the surface of the GA related nanoparticles. For this reason, Gum Arabic polymer layers helped to provide stable SERS signal and facilitated 2,4-D detection using Raman spectroscopy. It consists of polysaccharides rich in galactose and arabinose, and a small protein fraction associated with these polysaccharides, see SI Figure S10, and contains a large amount of -OH, $\mathrm{O}-$, and $-\mathrm{CH}$ bonds as well as a small portion of $-\mathrm{NH}_{2}$ and $-\mathrm{SH}$ group associated with the protein. ${ }^{41}$ Such functional groups may attract 2,4-D to the surface of the Ag-GA nano particles, through hydrogen bonding between -OH / -O- group on Ag-GA surface and the halogen $(-\mathrm{Cl})$ group in $2,4-\mathrm{D}$ moleculars, or $\pi-\pi$ interactions between $-\mathrm{CH}$ and the benzene ring of 2,4-D. ${ }^{42}$

In our study, 2,4-D was detected in water by dropping a 10 $\mu \mathrm{L}$ aliquot on top of Ag-GA SERS substrates. See Figure 3 (A) for a schematic of the SERS device setup. Typical Raman spectra, taken in deionized water and in a 1 pM 2,4-D solution, are shown for $\mathrm{S}_{4}$ in Figure 3 (B). Raman spectra of bare Ag - Gum Arabic SERS samples, acquired in the air, exhibited two broad peaks at $1400 \mathrm{~cm}^{-1}$ and $1601 \mathrm{~cm}^{-1}$ shown in SI Figure S11. Applying water onto the substrate did not change the spectral characteristics, but reduced the overall intensity, see also SI Figure $\mathrm{S} 11$. This could be attributed to the interaction between DI water and Gum Arabic which has numerous $-\mathrm{OH}$ bonds that have 
good affinity to water. After adding 2,4-D solution (1 pM) to a substrate, a distinct spectrum was observed after 30 minutes. Peaks $\sim 230 \mathrm{~cm}^{-1}$ and $\sim 1600 \mathrm{~cm}^{-1}$ gradually disappeared as the 2,4-D concentration increased, and new peaks at $293 \mathrm{~cm}^{-1}, 889$

(A)

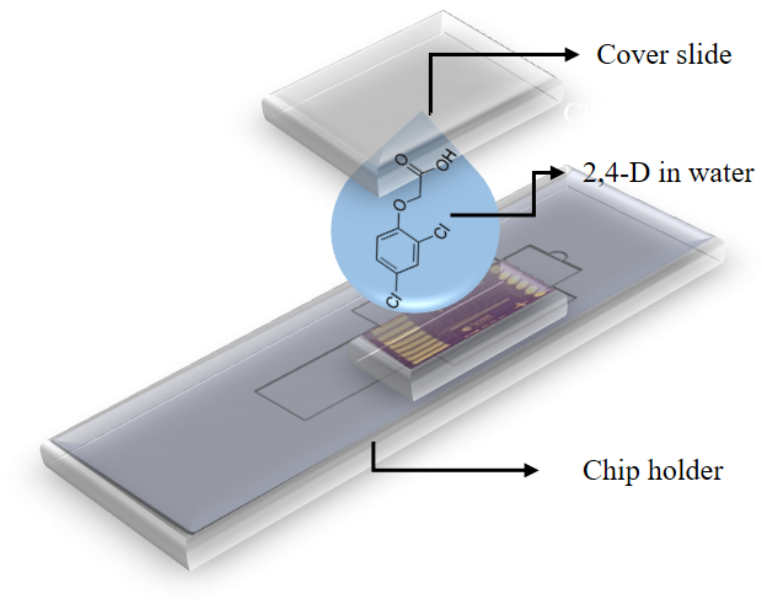

$\mathrm{cm}^{-1}$, peaks at $926 \mathrm{~cm}^{-1}, 1400 \mathrm{~cm}^{-1}$ and three sharp peaks around $1129 \mathrm{~cm}^{-1}$, appeared. In addition, we note that the 2,4-D solution did not dry during the course of our experiments.

(B)

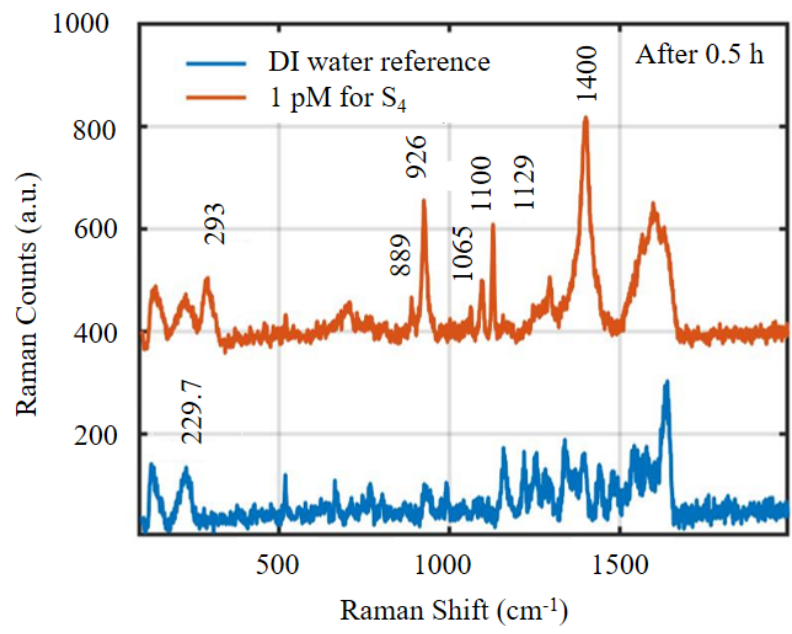

Figure 3 (A) Schematic illustration of the SERS measurement setup; (B) Spectrum of 2,4-D at 1 pM on structure four SERS substrate after a detection time of $30 \mathrm{~min}$ and spectrum of deionized water as the reference (background corrected). Spectra offset for clarity.

In the initial work, it was observed that structures $\mathrm{S}_{1}, \mathrm{~S}_{2}, \mathrm{~S}_{3}$ were able to detect $0.1 \mathrm{nM}(0.0221 \mathrm{ppb}) 2,4-\mathrm{D}$ in $30 \mathrm{~min}$; while structure $\mathrm{S}_{4}$ was able to detect as low as $1 \mathrm{pM}(0.221 \mathrm{ppt}) 2,4-$ $\mathrm{D}$ in the same time frame. An immediate response was observed for $0.1 \mathrm{nM} 2,4-\mathrm{D}$ ( $\mathrm{S}_{4}$ substrates) which is more than four times lower than the MRL (0.1 ppb) required by EU water framework directive for individual pesticides. ${ }^{43-44}$ As a result, structure $\mathrm{S}_{4}$ substrates were selected for further sensing studies reported below. The better sensing capabilities of $\mathrm{S}_{4}$ could be attributed to the morphology and size difference of the Ag-GA nano particles. ${ }^{45-47}$

\section{Linear calibration for 2,4-D detection in DI water}

The reproducibility for $\mathrm{S}_{4}(2,4-\mathrm{D}$ concentration at $2 \mu \mathrm{M})$ at 25 random points is shown in Figure 4 (A). The peak ratio $\mathrm{I}_{293}$ $\mathrm{cm}-1 / \mathrm{I}_{1400 \mathrm{~cm}-1}, \mathrm{I}_{1130 \mathrm{~cm}-1} / \mathrm{I}_{1400 \mathrm{~cm}-1}$ were studied as those peak ratios exhibited a good linear response towards different concentration of 2,4-D. The Relative Standard Deviation (RSD) for $\mathrm{I}_{293}$ $\mathrm{cm}-1 / \mathrm{I}_{1400 \mathrm{~cm}-1}, \mathrm{I}_{1130 \mathrm{~cm}-1} / \mathrm{I}_{1400 \mathrm{~cm}-1}$ were $9 \%$ and $11 \%$, respectively, see Figure 4 (B).

Different 2,4-D concentrations ranging from $0.2 \mathrm{mM}$ down to $1 \mathrm{pM}$ in DI water were used for the calibration study, see Figure $4(C)$. The peak ratios $I_{293 \mathrm{~cm}-1} / I_{1400 \mathrm{~cm}-1}$ and $I_{1130 \mathrm{~cm}-1} / I_{1400}$ $\mathrm{cm}-1$ increased with increasing 2,4-D concentration, while the broad peak near $1600 \mathrm{~cm}^{-1}$ decreased because of the overall coverage of 2,4-D molecules as the concentration increased. Figure 4 (D) showed the plot of $\mathrm{I}_{293 \mathrm{~cm}-1} / \mathrm{I}_{1400 \mathrm{~cm}-1}$ and $\mathrm{I}_{1130 \mathrm{~cm}-1} / \mathrm{I}_{1400 \mathrm{~cm}-1}$ against the logarithm of the concentrations of the 2,4-D solutions. Each spectrum was obtained by averaging ten spectra acquired from different random spots on the same substrate. In the range of 2,4-D concentration studied, a linear calibration plot of $\mathrm{y}=0.6296+0.0266 * \log _{10}(\mathrm{x})$, with an $\mathrm{R}^{2}=0.9530$ was obtained. The data did show variability; the relation between $\mathrm{I}_{293}$ $\mathrm{cm}-1 / \mathrm{I}_{1400 \mathrm{~cm}-1}(\mathrm{y})$ and $\log _{10}($ concentration $($ in $\mathrm{nM})$ ) $(\mathrm{x})$ was: $\mathrm{y}=$ $0.4078+0.0197 * \log _{10}(x), R^{2}=0.8990 ;$ which, with a low slope in the calibration curve, limits the accuracy in quantitative detection. However, we note that SERS sensors can be used to detect the presence of 2,4-D and trigger sample collection for further analysis in dedicated laboratories; or to determine the 2,4-D concentration by standard addition methods. This SERS platform offers a rapid approach to monitoring the presence of 2,4-D pesticide at ultra-low concentrations. Similar experiments were undertaken with Gum Arabic that was purchased from Amazon, as shown in SI figure S12 and exhibited similar results. 
(A)
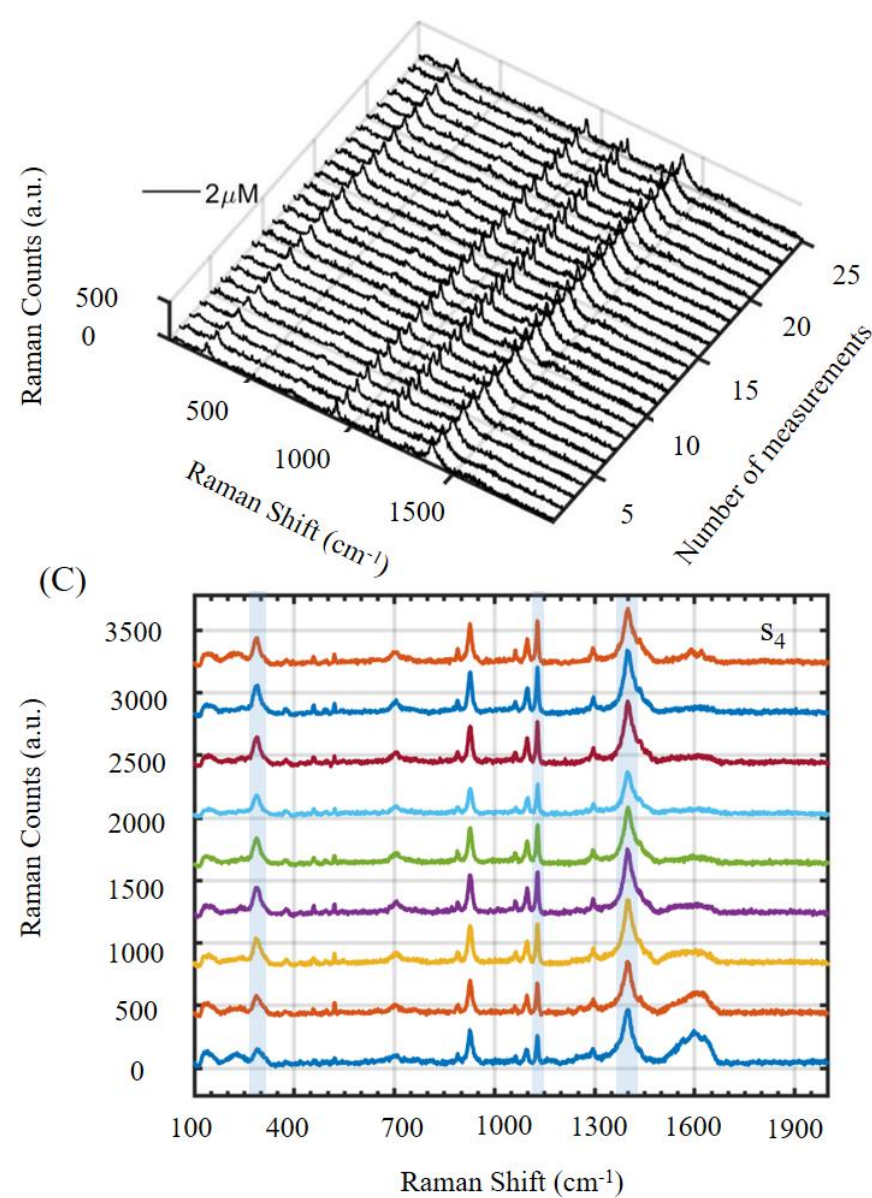

(B)
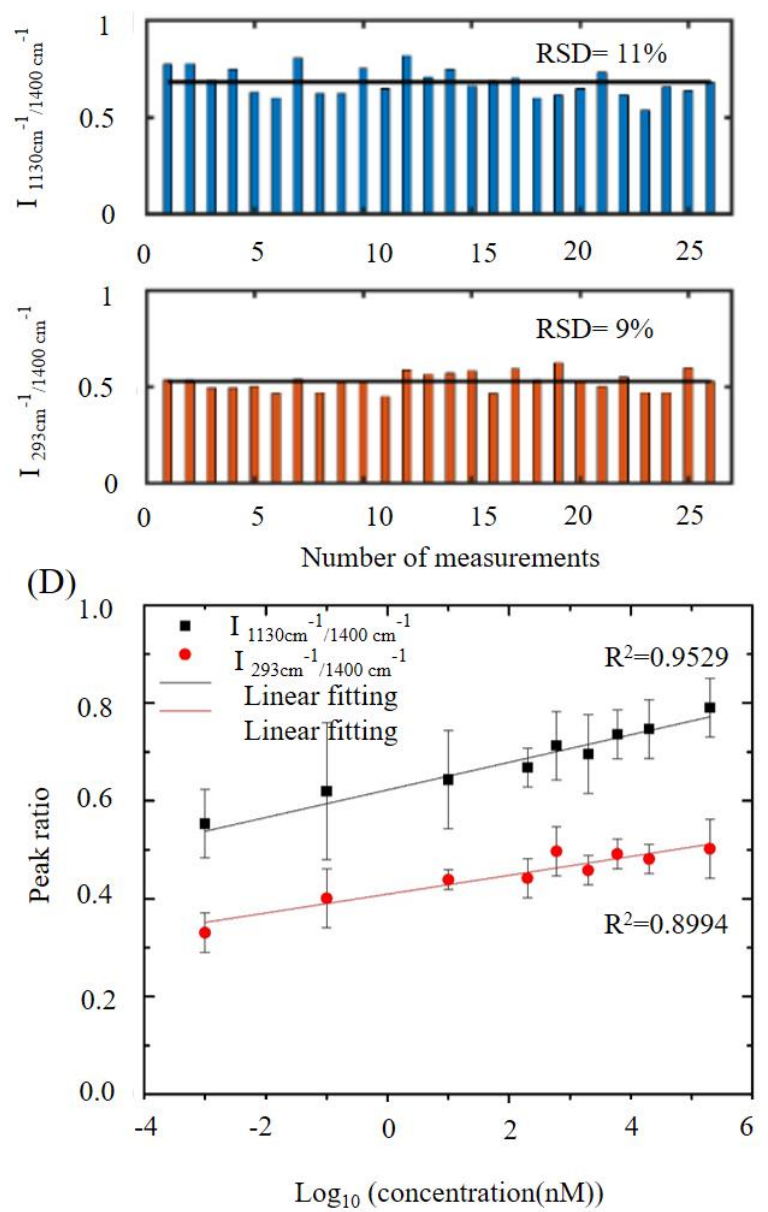

Figure 4 (A) Waterfall spectra of 25 random points for $2 \mu \mathrm{M}$ 2,4-D on $\mathrm{S}_{4}$ SERS substrate. (B) Histogram of $\mathrm{I}_{293} \mathrm{~cm}^{-1} / \mathrm{I}_{1400} \mathrm{~cm}^{-1}, \mathrm{I}_{1130} \mathrm{~cm}^{-1} /$

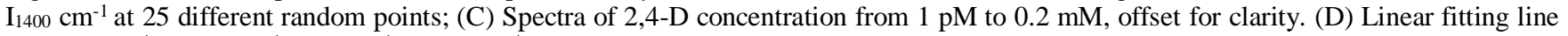

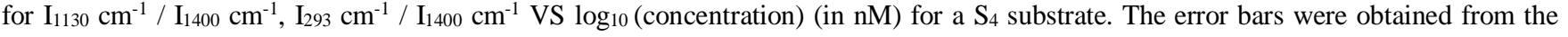
standard deviation (SD) of ten measurements at random points.

\section{Linear calibration for $2,4-\mathrm{D}$ detection in real samples}

The effect of different matrices on the SERS sensor performance was studied by spiking both commercial mineral water and river water samples with known 2,4-D concentrations. The mineral composition for the commercial mineral water is shown in table S3 in SI. Ten measurements were undertaken for each concentration on a substrate, the experiments were repeated three times on three individual SERS substrates. The error bar was calculated using data from the standard deviation (SD) of the three replicates across the three SERS substrates and show that the interaction of the sensor with 2,4-D in the presence of different ions remains largely unaffected.

The reference spectrum for mineral water is shown in Figure 5 (A), the interference peaks near $1130 \mathrm{~cm}^{-1}$ are probably due to the interaction between Gum Arabic and chemicals present in mineral water. In the range of concentration from $0.15 \mathrm{nM}$ to $1.5 \mu \mathrm{M}$, a linear calibration plot was obtained: $\mathrm{y}=0.5865+$ $0.0329 * \log _{10}(\mathrm{x})$, where $\mathrm{x}$ is the peak ratio $\mathrm{I}_{1130 \mathrm{~cm}-1} / \mathrm{I}_{1400 \mathrm{~cm}-1}$, with a $\mathrm{R}^{2}=0.9804$. Similar results were obtained for river water, see Figure 5 (B) again in the concentration range of 0.015 $\mathrm{nM}$ to $1.5 \mu \mathrm{M}$. A linear calibration plot of $\mathrm{y}=0.6456+$ $0.0180 * \log _{10}(\mathrm{x})$, with $\mathrm{R}^{2}=0.9681$ was obtained. A direct comparison of the sensor developed herein with those reported in the literature is provided in SI Table 2 and Table 3, as can be seen the senor performance in detection limit is better than previously reported SERS platforms ${ }^{12,14,19,21-23,26}$ and better than high-end laboratory based HPLC-MS instrumentation or other platforms ${ }^{43-44,48-57}$. 
(A)

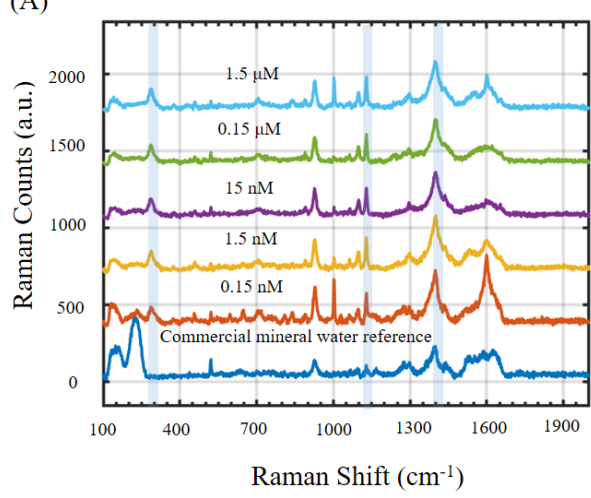

(B)

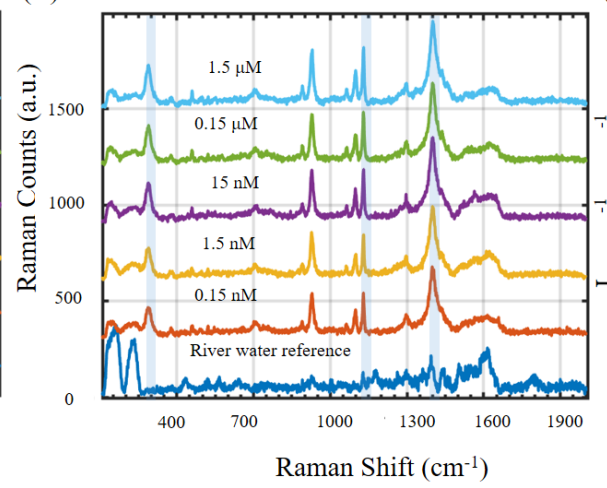

(C)

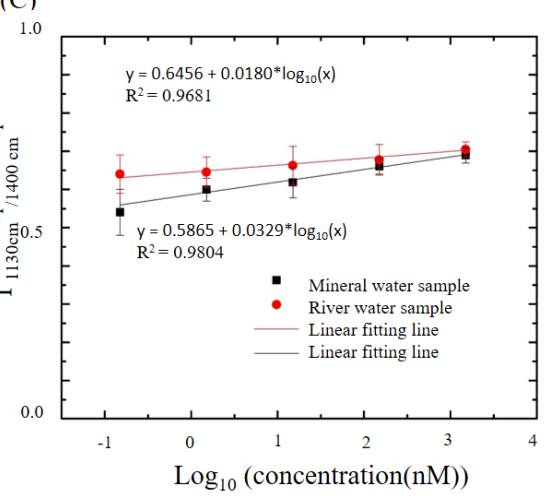

Figure 5 Spectra for real sample (A) commercial mineral water; (B) river water on $\mathrm{S}_{4}$ SERS substrates at concentration $0.15 \mathrm{nM}, 1.5 \mathrm{nM}, 15$ $\mathrm{nM}, 0.15 \mu \mathrm{M}, 1.5 \mu \mathrm{M}$, and the spectra for reference water samples, spectra offset for clarity. (C) Linear calibration lines for both real samples.

\section{CONCLUSIONS}

In summary, proof-of-concept large-area Ag-Gum Arabic nanocluster-based SERS substrates were prepared using an electrochemical deposition method. This fabrication method was environmental friendly, cost effective, simple, and rapid. These SERS substrates could detect 2,4-D pesticide in water directly without sample pre-treatment with a limit of detection 1 pM achieved. Detection of 2,4-D in relevant matrixes such as mineral or river water was also achieved down to $0.15 \mathrm{nM}$. Compared to previous 2,4-D detection study by SERS, see SI table S2, the present SERS platform was faster and was able to detect 2,4-D down to $1 \mathrm{pM}$. This was around 50 times lower than the lowest detection ability and three times lower than the calculated LOD reported by a previous SERS study. ${ }^{22}$ Work is now on-going to improve the SERS sensor performance using molecular imprint techniques ${ }^{20,54-56}$ to optimize trapping of target analytes.

\section{ASSOCIATED CONTENT}

\section{SUPPORTING INFORMATION.}

This material is available free of charge via the Internet at http://pubs.acs.org.

SI includes figures about chip fabrication process, electrochemical deposition setup, chip circuit design, saccharides structure in Gum Arabic, SEM images for SERS substrate optimization, current-time plot and optical images for Ag-GA SERS substrate deposition, SERS spectra for Ag-GA bare substrate in air and in DI water, data process by Matlab, the linear calibration for commercial Gum Arabic-Ag SERS substrate, tables for the literature comparison, table for the mineral water composition.

\section{AUTHOR INFORMATION}

\section{Corresponding Author}

Pierre Lovera-Nanotechnology Group, Tyndall National Institute University College Cork, Dyke Parade, Cork, Ireland Eamil: pierre.lovera@tyndall.ie

\section{Authors}

Yuqing Yang-Nanotechnology Group, Tyndall National Institute University College Cork, Dyke Parade, Cork, Ireland Eamil: yuqing.yang@tyndall.ie

Alan O'Riordan- Nanotechnology Group, Tyndall National Institute - University College Cork, Dyke Parade, Cork, Ireland Eamil: alan.oriordan@tyndall.ie

\section{Author Contributions}

Y.Y. undertake experimental results including the Raman data analysis and manuscript preparation. P.L. responsible for supervising and data analysis \& manuscript preparation and A. O'R. responsible for supervising and manuscript preparation.

\section{ACKNOWLEDGMENT}

This work has received funding from the European Union's Horizon 2020 research and innovation programme under the Marie Skłodowska-Curie grant agreement No: H2020-MSCA-ITN-2018813680 . This work has been supported in part by a research grant for the VistaMilk Centre Science Foundation Ireland (SFI); Department of Agriculture, Food and the Marine (DAFM) under Grant Number $16 / \mathrm{RC} / 3835$ 


\section{REFERENCES}

1. https://population.un.org/wpp/Download/Standard/Population/.

2. Ju, Z.; Liu, S.-S.; Xu, Y.-Q.; Li, K., Combined Toxicity of 2,4-Dichlorophenoxyacetic Acid and Its Metabolites 2,4-Dichlorophenol (2,4DCP) on Two Nontarget Organisms. ACS omega 2019, 4 (1), 1669-1677.

3. Carles, L.; Martin-Laurent, F.; Devers, M.; Spor, A.; Rouard, N.; Beguet, J.; Besse-Hoggan, P.; Batisson, I., Potential of preventive bioremediation to reduce environmental contamination by pesticides in an agricultural context: A case study with the herbicide 2,4-D. Journal of Hazardous Materials 2021, 416, 125740.

4. Prado, A. G. S.; Airoldi, C., Effect of the pesticide 2,4-D on microbial activity of the soil monitored by microcalorimetry. Thermochimica Acta 2000, 349 (1), 17-22.

5. Health Service Executive, N. D. W. G., Pesticides in Drinking Water

Frequently Asked Questions. 2018.

6. Meftaul, I. M.; Venkateswarlu, K.; Dharmarajan, R.; Annamalai, P.; Megharaj, M., Movement and Fate of 2,4-D in Urban Soils: A Potential Environmental Health Concern. ACS Omega 2020, 5 (22), 13287-13295.

7. Organization, W. H., 2,4-D in Drinking-water

Background document for development of

WHO Guidelines for Drinking-water Quality. 2003.

8. Meulenberg, E. P.; Stoks, P. G., Water quality control in the production of drinking water from river water. The application of immunological techniques for the detection of chlorophenoxy acid herbicides (2,4-D). Analytica Chimica Acta 1995, 311 (3), 407-413.

9. Congur, G., Monitoring of glyphosate-DNA interaction and synergistic genotoxic effect of glyphosate and 2,4-dichlorophenoxyacetic acid using an electrochemical biosensor. Environmental Pollution 2021, 271, 116360.

10. Kumar, S.; Mehta, D.; Chaudhary, S.; Chaudhary, G. R., Pr@Gd2O3 nanoparticles: An effective fluorescence sensor for herbicide 2,4dichlorophenoxyacetic acid. Journal of Molecular Liquids 2021, 324, 114712.

11. El-Maghrabey, M.; Suzuki, H.; Kishikawa, N.; Kuroda, N., A sensitive chemiluminescence detection approach for determination of 2,4dinitrophenylhydrazine derivatized aldehydes using online UV irradiation - luminol CL reaction. Application to the HPLC analysis of aldehydes in oil samples. Talanta 2021, 233, 122522.

12. Xu, Y.; Kutsanedzie, F. Y. H.; Hassan, M. M.; Zhu, J.; Li, H.; Chen, Q., Functionalized hollow Au@ Ag nanoflower SERS matrix for pesticide sensing in food. Sensors and Actuators B: Chemical 2020, 324, 128718.

13. Langer, J.; Jimenez de Aberasturi, D.; Aizpurua, J.; Alvarez-Puebla, R. A.; Auguié, B.; Baumberg, J. J.; Bazan, G. C.; Bell, S. E. J.; Boisen, A.; Brolo, A. G.; Choo, J.; Cialla-May, D.; Deckert, V.; Fabris, L.; Faulds, K.; García de Abajo, F. J.; Goodacre, R.; Graham, D.; Haes, A. J.; Haynes, C. L.; Huck, C.; Itoh, T.; Käll, M.; Kneipp, J.; Kotov, N. A.; Kuang, H.; Le Ru, E. C.; Lee, H. K.; Li, J. -F.; Ling, X. Y.; Maier, S. A.; Mayerhöfer, T.; Moskovits, M.; Murakoshi, K.; Nam, J.-M.; Nie, S.; Ozaki, Y.; Pastoriza-Santos, I.; Perez-Juste, J.; Popp, J.; Pucci, A.; Reich, S.; Ren, B.; Schatz, G. C.; Shegai, T.; Schlücker, S.; Tay, L.-L.; Thomas, K. G.; Tian, Z.-Q.; Van Duyne, R. P.; Vo-Dinh, T.; Wang, Y.; Willets, K. A.; Xu, C.; Xu, H.; Xu, Y.; Yamamoto, Y. S.; Zhao, B.; Liz-Marzán, L. M., Present and Future of Surface-Enhanced Raman Scattering. ACS Nano 2020, 14 (1), $28-117$.

14. Mao, P.; Liu, C.; Favraud, G.; Chen, Q.; Han, M.; Fratalocchi, A.; Zhang, S., Broadband single molecule SERS detection design ed by warped optical spaces. Nature Communications 2018, 9 (1), 5428.

15. Zhu, W.; Crozier, K. B., Quantum mechanical limit to plasmonic enhancement as observed by surface-enhanced Raman scattering. Nature Communications 2014, 5 (1), 5228

16. Zhang, Y.; Gu, Y.; He, J.; Thackray, B. D.; Ye, J., Ultrabright gap-enhanced Raman tags for high-speed bioimaging. Nature Communications 2019, $10(1), 3905$.

17. Liu, W.; Bai, H.; Li, X.; Li, W.; Zhai, J.; Li, J.; Xi, G., Improved Surface-Enhanced Raman Spectroscopy Sensitivity on Metallic Tungsten Oxide by the Synergistic Effect of Surface Plasmon Resonance Coupling and Charge Transfer. The Journal of Physical Chemistry Letters 2018, 9 (14), 4096-4100.

18. Creedon, N.; Lovera, P.; Moreno, J. G.; Nolan, M.; O’Riordan, A., Highly Sensitive SERS Detection of Neonicotinoid Pesticides. Complete Raman Spectral Assignment of Clothianidin and Imidacloprid. The Journal of Physical Chemistry A 2020, 124 (36), $7238-7247$.

19. Hassan, M. M.; Jiao, T.; Ahmad, W.; Yi, X.; Zareef, M.; Ali, S.; Li, H.; Chen, Q., Cellulose paper-based SERS sensor for sensitive detection of 2,4-D residue levels in tea coupled uninformative variable elimination-partial least squares. Spectrochimica Acta Part A: Molecular and Biomolecular Spectroscopy 2021, 248, 119198.

20. Hua, M. Z.; Feng, S.; Wang, S.; Lu, X., Rapid detection and quantification of 2,4-dichlorophenoxyacetic acid in milk using molecularly imprinted polymers-surface-enhanced Raman spectroscopy. Food Chemistry 2018, 258, 254-259.

21. Hassan, M. M.; Zareef, M.; Jiao, T.; Liu, S.; Xu, Y.; Viswadevarayalu, A.; Li, H.; Chen, Q., Signal optimized rough silver nanoparticle for rapid SERS sensing of pesticide residues in tea. Food Chemistry 2021, 338, 127796.

22. Xu, Y.; Kutsanedzie, F. Y. H.; Hassan, M.; Zhu, J.; Ahmad, W.; Li, H.; Chen, Q., Mesoporous silica supported orderly-spaced gold nanoparticles SERS-based sensor for pesticides detection in food. Food Chemistry 2020, 315, 126300.

23. Joseph, D.; Kwak, C. H.; Huh, Y. S.; Han, Y.-K., Synthesis of AuAg@Ag core@ shell hollow cubic nanostructures as SERS substrates for attomolar chemical sensing. Sensors and Actuators B: Chemical 2019, 281, 471-477.

24. Li, Z. H.; Bai, J. H.; Zhang, X.; Lv, J. M.; Fan, C. S.; Zhao, Y. M.; Wu, Z. L.; Xu, H. J., Facile synthesis of Au nanoparticle-coated Fe3O4 magnetic composite nanospheres and their application in SERS detection of malachite green. Spectrochimica Acta Part A: Molecular and Biomolecular Spectroscopy 2020, 241, 118532.

25. Petti, L.; Capasso, R.; Rippa, M.; Pannico, M.; La Manna, P.; Peluso, G.; Calarco, A.; Bobeico, E.; Musto, P., A plasmonic na nostructure fabricated by electron beam lithography as a sensitive and highly homogeneous SERS substrate for bio-sensing applications. Vibrational Spectroscopy 2016, 82, 22-30.

26. Shi, G.; Wang, M.; Zhu, Y.; Yan, X.; Pan, S.; Zhang, A., Nanoflower-like Ag/AAO SERS platform with quasi-photonic crystal nanostructure for efficient detection of goat serum. Current Applied Physics 2019, 19 (11), 1276-1285.

27. Creedon, N. C.; Lovera, P.; Furey, A.; O'Riordan, A., Transparent polymer-based SERS substrates templated by a soda can. Sensors and Actuators B: Chemical 2018, 259, 64-74.

28. Lovera, P.; Creedon, N.; Alatawi, H.; Mitchell, M.; Burke, M.; Quinn, A. J.; O'Riordan, A., Low-cost silver capped polystyrene nanotube arrays as super-hydrophobic substrates for SERS applications. Nanotechnology 2014, 25 (17), 175502.

29. Street, C. A.; Anderson, D. M. W., Refinement of structures previously proposed for gum arabic and other acacia gum exudates. Talanta 1983, $30(11), 887-893$. 
30. Goff, H. D.; Guo, Q., Chapter 1 The Role of Hydrocolloids in the Development of Food Structure. In Handbook of Food Structure Development, The Royal Society of Chemistry: 2020; pp 1-28.

31. Sanchez, L. M.; Ollier, R. P.; Pereira, A. E. S.; Fraceto, L. F.; Alvarez, V. A., Chapter 15 - Pesticide removal from industrial effluents using biopolymeric materials. In Biopolymer Membranes and Films, de Moraes, M. A.; da Silva, C. F.; Vieira, R. S., Eds. Elsevier: 2020; pp 359382 .

32. Kee, Y. L.; Mukherjee, S.; Pariatamby, A., Effective remediation of phenol,2,4-bis(1,1-dimethylethyl) and bis(2-ethylhexyl) phthalate in farm effluent using Guar gum - A plant based biopolymer. Chemosphere 2015, 136, 111-117.

33. Chen, Z.; Ye, H.; Chen, Y.; Xu, J., Tuning the dispersity and sphericity of silver particles by wrapping mechanism using Arab ic gum. Materials Letters 2021, 293, 129665.

34. Anwar, A.; Masri, A.; Rao, K.; Rajendran, K.; Khan, N. A.; Shah, M. R.; Siddiqui, R., Antimicrobial activities of green synthesized gumsstabilized nanoparticles loaded with flavonoids. Scientific Reports 2019, 9 (1), 3122.

35. Song, J. E.; Phenrat, T.; Marinakos, S.; Xiao, Y.; Liu, J.; Wiesner, M. R.; Tilton, R. D.; Lowry, G. V., Hydrophobic Interactions Increase Attachment of Gum Arabic- and PVP-Coated Ag Nanoparticles to Hydrophobic Surfaces. Environmental Science \& Technology 2011, 45 (14), 59885995 .

36. Seymour, I.; O'Sullivan, B.; Lovera, P.; Rohan, J. F.; O'Riordan, A., Elimination of Oxygen Interference in the Electrochemical Detection of Monochloramine, Using In Situ pH Control at Interdigitated Electrodes. ACS Sensors 2021, 6 (3), 1030-1038.

37. Schulze, H. G.; Foist, R. B.; Okuda, K.; Ivanov, A.; Turner, R. F. B., A Small-Window Moving Average-Based Fully Automated Baseline Estimation Method for Raman Spectra. Appl. Spectrosc. 2012, 66 (7), 757-764.

38. Daly, R.; Narayan, T.; Shao, H.; O’Riordan, A.; Lovera, P., Platinum-Based Interdigitated Micro-Electrode Arrays for Reagent-Free Detection of Copper. Sensors 2021, 21 (10).

39. Guler, M. T.; Bilican, I., Capacitive detection of single bacterium from drinking water with a detailed investigation of electrical flow cytometry. Sensors and Actuators A: Physical 2018, 269, 454-463.

40. D Bhakat, P. B. A. B., Electrical conductive properties of some composites of gum arabic bioplolymer and magnetite nanoparticles. Indian Journal of Pure \& Applied Physics 2018, 56, 428-433.

41. Mariod, A. A., 6 - Chemical Properties of Gum Arabic. In Gum Arabic, Mariod, A. A., Ed. Academic Press: 2018 ; pp 67-73.

42. Schneider, H.-J., Dispersive Interactions in Solution Complexes. Accounts of Chemical Research 2015, 48 (7), $1815-1822$

43. Communities, E., EU Policy for a sustainable use of pesticides

The story behind the Strategy. 2007.

44. establishing a framework for Community action in the field of water policy. Official Journal of the European Communities 2000

45. Stamplecoskie, K. G.; Scaiano, J. C.; Tiwari, V. S.; Anis, H., Optimal Size of Silver Nanoparticles for Surface-Enhanced Raman Spectroscopy. The Journal of Physical Chemistry C 2011, 115 (5), 1403-1409.

46. Shen, X. S.; Wang, G. Z.; Hong, X.; Zhu, W., Nanospheres of silver nanoparticles: agglomeration, surface morphology control and application as SERS substrates. Physical Chemistry Chemical Physics 2009, 11 (34), 7450-7454.

47. Joseph, D.; Huh, Y. S.; Han, Y.-K., A top-down chemical approach to tuning the morphology and plasmon resonance of spiky nanostars for enriched SERS-based chemical sensing. Sensors and Actuators B: Chemical 2019, 288, 120-126.

48. de Amarante, O. P., Jr.; Brito, N. M.; Dos Santos, T. C.; Nunes, G. S.; Ribeiro, M. L., Determination of 2,4-dichlorophenoxyacetic acid and its major transformation product in soil samples by liquid chromatographic analysis. Talanta 2003, 60 (1), 115-21.

49. Feng, X.; Zhang, G.; Chin, L. K.; Liu, A. Q.; Liedberg, B., Highly Sensitive, Label-Free Detection of 2,4-Dichlorophenoxyacetic Acid Using an Optofluidic Chip. ACS Sensors 2017, 2 (7), 955-960.

50. Hassan, M. M.; Jiao, T. H.; Ahmad, W.; Yi, X.; Zareef, M.; Ali, S.; Li, H. H.; Chen, Q. S., Cellulose paper-based SERS sensor for sensitive detection of 2,4-D residue levels in tea coupled uninformative variable elimination-partial least squares. SPECTROCHIMICA ACTA PART AMOLECULAR AND BIOMOLECULAR SPECTROSCOPY 2021, 248.

51. Kormosh, Z. A.; Zhurba, E. S.; Antal, I. P.; Kormosh, A. Z.; Bazel, Y. R., Spectrophotometric Determination of 2,4-Dichlorophenoxyacetic Acid Using Extraction with Astrafloxin. JOURNAL OF ANALYTICAL CHEMISTRY 2020, 75 (7), 909-912.

52. Liu, F. P.; Xu, Q.; Huang, W.; Zhang, Z. F.; Xiang, G.; Zhang, C. Z.; Liang, C. Y.; Lian, H.; Peng, J. Y., Green synthesis of porous graphene and its application for sensitive detection of hydrogen peroxide and 2,4-dichlorophenoxyacetic acid. ELECTROCHIMICA ACTA 2019, $295,615-623$

53. Orooji, N.; Takdastan, A.; Yengejeh, R. J.; Jorfi, S.; Davami, A. H., A quick and inexpensive method to determine 2,4dichlorophenoxyacetic acid residues in water samples by HPLC. DESALINATION AND WATER TREATMENT 2021, 217, 329-338.

54. Peng, D. H.; Li, X.; Zhang, L. Z.; Gong, J. M., Novel visible-light-responsive photoelectrochemical sensor of 2,4-dichlorophenoxyacetic acid using molecularly imprinted polymer/BiOI nanoflake arrays. ELECTROCHEMISTRY COMMUNICATIONS 2014, 47, 9-12.

55. Shi, H. J.; Zhao, G. H.; Liu, M. C.; Zhu, Z. L., A novel photoelectrochemical sensor based on molecularly imprinted polymer modified $\mathrm{TiO} 2$ nanotubes and its highly selective detection of 2,4-dichlorophenoxyacetic acid. ELECTROCHEMISTRY COMMUNICATIONS 2011, 13 (12), 1404-1407.

56. Xie, C.; Gao, S.; Guo, Q.; Xu, K., Electrochemical sensor for 2,4-dichlorophenoxy acetic acid using molecularly imprinted polypyrrole membrane as recognition element. Microchimica Acta 2010, 169 (1), 145-152.

57. Zhang, Z.; Ma, X.; Li, B.; Zhao, J.; Qi, J.; Hao, G.; Jianhui, R.; Yang, X., Fluorescence detection of 2,4-dichlorophenoxyacetic acid by ratiometric fluorescence imaging on paper-based microfluidic chips. Analyst 2020, 145 (3), 963-974. 